\title{
Sodium-Glucose Cotransporter-2 Inhibitors (SGLT-2i) Reduce Hospitalization for Heart Failure Only and Have No Effect on Atherosclerotic Cardiovascular Events: A Meta-Analysis
}

Binayak Sinha $\cdot$ Samit Ghosal (D)

Received: February 2, 2019 / Published online: March 14, 2019

(C) The Author(s) 2019

\section{ABSTRACT}

Introduction: Although the positive effects of sodium-glucose cotransporter-2 inhibitors (SGLT-2i) on hospitalization for heart failure in type 2 diabetes (T2D) seem definite, some doubt exists about their effects on atherosclerotic cardiovascular disease (ASCVD). This study aims to shed light on this debatable issue.

Methods: An electronic database search (Cochrane Library, PubMed and Embase) was performed using two groups of terms ["sodium glucose cotransporter2 inhibitor", "dapagliflozin", "canagliflozin", "empagliflozin", "ertugliflozin"] AND ["major adverse cardiac events", "MACE", "cardiovascular death or hospitalization for heart failure", non-fatal myocardial infarction", "non-fatal stroke", "cardiovascular death", "hospitalization for heart failure"] and the cardiovascular outcome trials (CVOT) and pre-approval studies in phase 3 of all the SGLT2i analysed using comprehensive meta-analysis (CMA) software, version 3, Biostat Inc., Englewood, NJ, USA.

Enhanced Digital Features To view enhanced digital features for this article go to https://doi.org/10.6084/ m9.figshare.7813982.

B. Sinha

AMRI Hospitals, Kolkata, India

S. Ghosal ( $\square)$

Nightingale Hospital, Kolkata, India

e-mail: ramdasghosal@gmail.com
Results: Analysis of the CVOT revealed that the hazard ratio of the pooled effect size for MACE was statistically significant (HR $0.89,95 \%$ CI $0.83-0.96, P=0.002$ ). There was a significant reduction in non-fatal myocardial infarction (MI) (HR 0.87, 95\% CI 0.78-0.97, $P=0.01$ ), but no improvement was seen for non-fatal stroke (HR 1.01, 95\% CI 0.89-1.16, $P=0.83$ ). The pooled analysis of this end point showed statistically significant reduction of the composite of $\mathrm{CV}$ death or hospitalization for heart failure (hHF) (HR 0.76, 95\% CI 0.67-0.87, $P<0.001$ ) and hHF (HR 0.69, 95\% CI 0.61-0.79, $P<0.001$ ), but not for CV death alone (HR 0.82, $95 \%$ CI $0.64-1.05, P=0.11)$. The meta-analysis of the events in the pooled analysis of the phase 3 trials reveals that the hazard ratio for MACE was statistically nonsignificant (HR 0.83, 95\% CI $0.66-1.03, P=0.10)$. There was a $34 \%$ statistically significant reduction in MI $(95 \% \mathrm{CI}$ $0.48-0.91, P=0.01$ ), a $36 \%$ statistically significant reduction in CV death (95\% CI 0.41-0.97, $P=0.04)$ and a $64 \%$ statistically significant reduction in hHF (95\% CI 0.18-0.69, $P<0.01)$. In contrast, there was a $17 \%$ statistically nonsignificant increased risk of stroke $(95 \%$ CI 0.80-1.70, $P=0.40$ ).

Conclusion: The predominant impact of SGLT$2 \mathrm{i}$ is on "hHF or CV mortality" composite driven predominantly by reduction in hHF and not atherosclerotic CV disease. 
Keywords: Cardiovascular death; Hospitalization for heart failure; Major cardiovascular events; Meta-analysis; Sodium glucose cotransporter 2 inhibitor; Type 2 diabetes

$\begin{array}{ll}\text { Abbreviations } \\ \text { ASCVD } & \text { Atherosclerotic cardiovascular disease } \\ \text { BP } & \text { Blood pressure } \\ \text { CV } & \text { Cardiovascular } \\ \text { CVOT } & \text { Cardiovascular outcome trial } \\ \text { hHF } & \text { Hospitalization for heart failure } \\ \text { MACE } & \text { Major adverse cardiac events } \\ \text { MI } & \text { Myocardial infarction } \\ \text { T2D } & \text { Type 2 diabetes }\end{array}$

\section{INTRODUCTION}

Sodium-glucose cotransporter-2 inhibitors (SGLT-2i) cause natriuresis and this leads to a reduction in extracellular fluid volume [1], which leads to a blood pressure reduction of 4-5 mm/1-2 mmHg. This reduction in blood pressure is not accompanied by an increase in heart rate [2]. Pre-clinical studies also have reported heart tissue remodelling after the administration of SGLT-2 inhibitors in association with a marked reduction of interstitial fibrosis [3]. These findings have previously been seen with mineralocorticoid receptor antagonists, which have similar properties and reduce heart failure and cardiovascular death [4].

All the CVOTs published [5-7] are in keeping with the above findings and suggest that the main beneficial effect of SGLT-2i is in reducing heart failure, except the EMPA REG outcome study [5], which showed a statistically significant reduction in $\mathrm{CV}$ death. However, the recent meta-analysis on the primary (preventative) and secondary effects of the sodium-glucose cotransporter-2 inhibitors (SGLT-2i) on cardiovascular and renal outcomes published in The Lancet in November 2018 expands the boundaries of our understanding of SGLT-2i, but probably overemphasizes the salutary effects of SGLT-2i [8]. This meta-analysis indicates that the benefits of SGLT-2i extend from hospitalization for heart failure (hHF) to a reduction in atherosclerotic events and cardiovascular (CV) death. This is debatable and unconfirmed.

We therefore conducted a meta-analysis of the three selected cardiovascular outcomes trials (CVOTs) along with their supplementary materials and in-depth analysis of these studies by the regulatory bodies (FDA and EAMA) to assess the effects of these drugs on atherosclerotic events, CV death and hHF. We also pooled the events from all the phase 3 studies with all the available gliflozins and conducted a metaanalysis of the cardiovascular and hHF events in those studies. In addition, we also looked at the mechanistic data available on SGLT-2i to decipher and establish whether the beneficial effects of these molecules extend to ASCVD or are restricted only to hHF.

\section{METHODS}

\section{Selection Criteria}

An electronic database search was performed using the two groups of terms ["sodium glucose cotransporter 2 inhibitor", "dapagliflozin", "canagliflozin", "empagliflozin", "ertugliflozin"] AND ["Major Adverse Cardiac Events", "MACE", "cardiovascular death or hospitalization for heart failure", "non-fatal myocardial infarction", "non-fatal stroke", "cardiovascular death", "hospitalization for heart failure"]. Combining the two group of searches yielded 87 citations from Cochrane Library, PubMed and Embase databases (Fig. 1). Applying additional filters (RCTs, CVOTs) and a pre-specified inclusion criterion (studies with $>1000$ patients, duration of follow-up $\geq 1$ year and MACE as a primary end point) resulted in three citations available for analysis [5-7]. The preliminary pre-approval studies in phase 3 of all the SGLT-2i were also analyzed. This resulted in four citations fulfilling the criteria for evaluation [9-12]. As a result, there were two different cohorts taken up for the meta-analysis.

This meta-analysis was conducted on a pooled patient population of 34,322 in the CVOT arm and 23,334 in the phase 3 pooled analysis arm, using comprehensive meta-analysis (CMA) software version 3, Biostat Inc., 


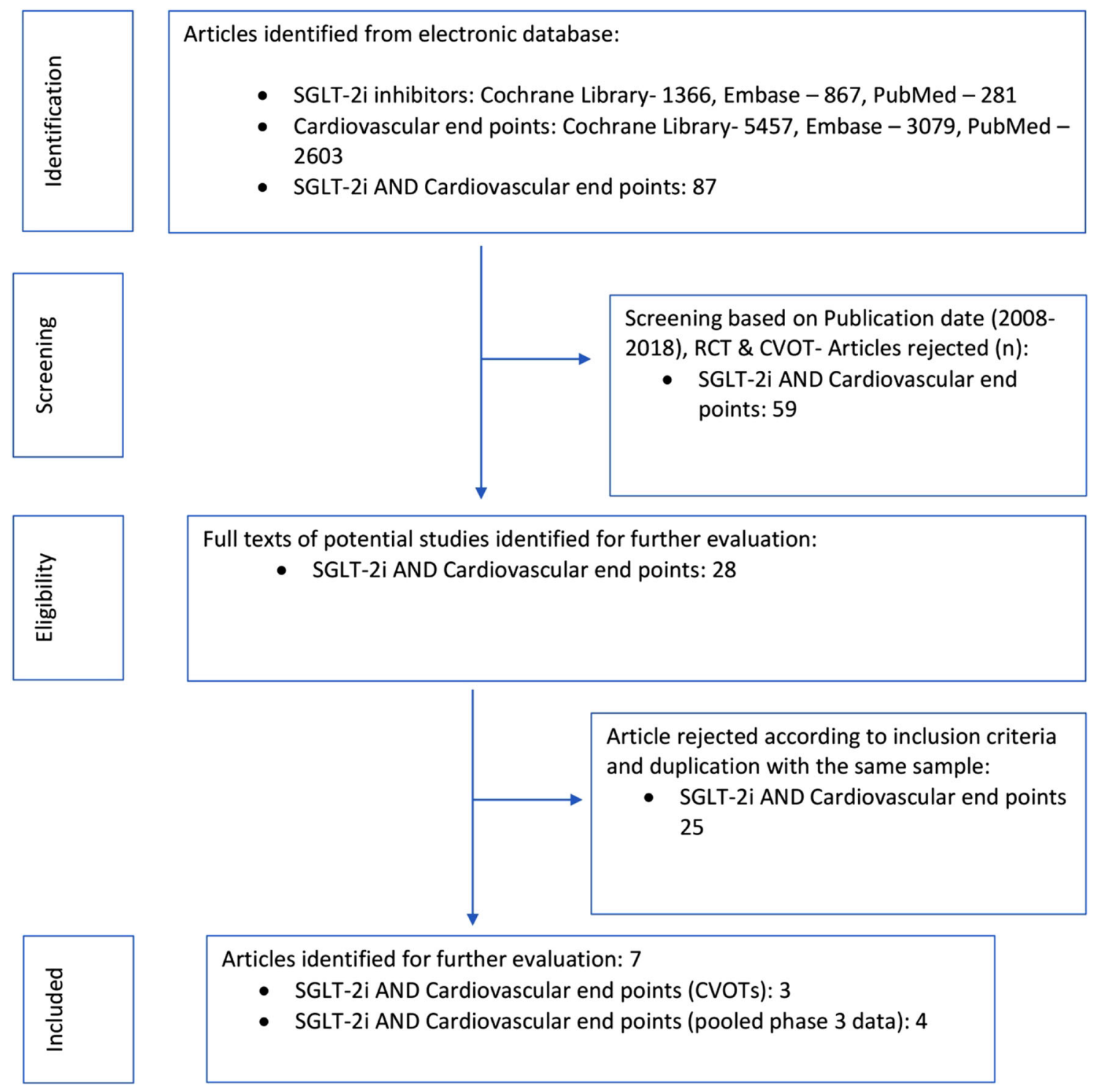

Fig. 1 Study selection process

Englewood, NJ, USA. Heterogeneity was assessed using the Cochrane $Q$ and Higgin's $I^{2}$ test and publication bias was assessed by funnel plots.

Depending on the presence or degree of heterogeneity $(<45 \%$ low, $45-75 \%$ moderate and $>75 \%$ high) and the study characteristics, a fixed or random effect model to assess the effect size was selected. The individual study bias was assessed using the Cochrane collaboration tool (Fig. 2). This article is based on previously conducted studies and does not contain any studies with human participants or animals performed by any of the authors.

\section{RESULTS}

\section{The CVOT Cohort}

The hazard ratio of the pooled effect size for major adverse cardiac events (MACE) was statistically significant (HR 0.89, 95\% CI 0.83-0.96, $P=0.002$ ) (Fig. 3a). There was a 


\begin{tabular}{|c|c|c|c|c|c|c|c|}
\hline \multicolumn{8}{|l|}{ CVOTs } \\
\hline EMPA REG & + & + & + & $?$ & - & + & + \\
\hline CANVAS Program & + & + & + & + & - & + & + \\
\hline DECLARE-TIMI 58 & + & + & + & + & - & + & + \\
\hline \multicolumn{8}{|l|}{$\begin{array}{l}\text { Phase } 3 \text { Pooled } \\
\text { data }\end{array}$} \\
\hline Empa & + & + & + & + & + & + & ? \\
\hline Dapa & + & + & + & + & + & + & ? \\
\hline Cana & + & + & + & + & + & + & ? \\
\hline Sonesson et al & + & + & + & + & ? & + & ? \\
\hline
\end{tabular}

\begin{tabular}{|c|c|c|c|c|c|}
\hline 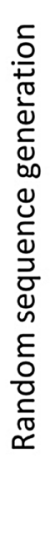 & 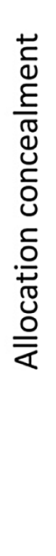 & 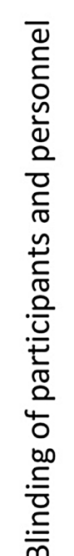 & 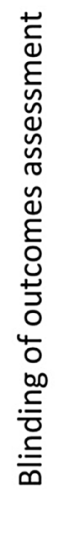 & 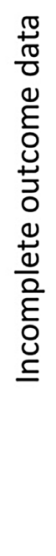 & 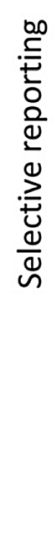 \\
\hline
\end{tabular}

Fig. 2 Quality assessment of studies. + Low risk of bias, ? unknown risk of bias, - high risk of bias

significant reduction in non-fatal MI (HR 0.87, 95\% CI $0.78-0.97, P=0.01$ ) but no improvement was seen for non-fatal stroke (HR 1.01, 95\% CI 0.89-1.16, $P=0.83$ ) (Fig. 3c, d).

DECLARE-TIMI 58 studied a primary end point different from the other CVOTs. This was the co-primary composite of "cardiovascular death or hospitalization for heart failure". The pooled analysis of this end point showed statistically significant reduction of the composite (HR 0.76, 95\% CI 0.67-0.87, $P<0.001$ ) (Fig. 3e).

Analyzing the individual end points however indicates that this composite superiority was exclusively driven by the hospitalization for the heart failure component (HR 0.69, 95\% CI $0.61-0.79, P<0.001)$ and not by $\mathrm{CV}$ death (HR $0.82,95 \%$ CI $0.64-1.05, P=0.11$ ) (Fig. 3b, f)

\section{The Phase 3 Pooled Analysis Cohort}

The hazard ratio for MACE was statistically nonsignificant (HR 0.83, 95\% CI 0.66-1.03, $P=0.10)$. However, there was a $34 \%$ statistically significant reduction in MI (95\% CI 0.48-0.91, $P=0.01)$, a $36 \%$ statistically significant reduction in $\mathrm{CV}$ death (95\% CI 0.41-0.97, $P=0.04$ ) and a $64 \%$ statistically significant reduction in hHF (95\% CI $0.18-0.69, P<0.01)$. In contrast, there was a $17 \%$ statistically nonsignificant increased risk of stroke (95\% CI $0.80-1.70$, $P=0.40)$.

\section{DISCUSSION}

Previous meta-analyses of CVOTs with SGLT-2i have deified this group of drugs, justifiably [8]. Although the reduction in hHF is no doubt reproducible and consistent, detailed dissection of other end points does not show that consistency across all the data analyzed (Fig. 4).

\section{Evidence from the CVOTs}

Contrary to the findings of the meta-analysis by Zelniker et al. [8], this meta-analysis (CVOT 


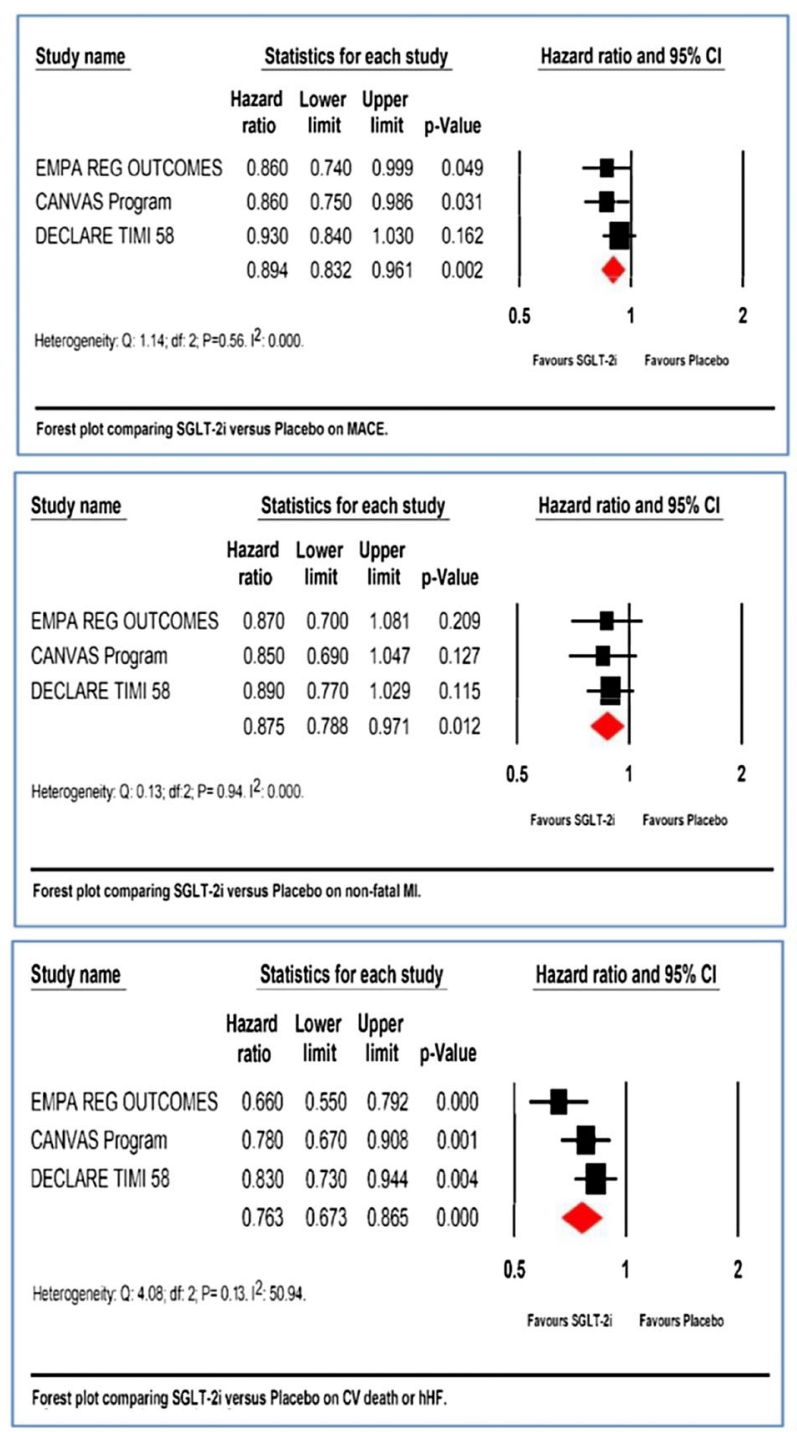

Fig. 3 Forest plot comparing SGLT-2i versus placebo on cardiovascular end points. a MACE; b CV death; c nonfatal MI; d non-fatal stroke; e CV death or hHF; f hHF.

cohort) indicates that though there is a reduction in non-fatal MI, this signal is not replicated in non-fatal stroke. This would suggest that the benefit documented in the earlier meta-analysis in reducing atherosclerotic events is inconsistent and must be analyzed with caution.

Moreover, both the CANVAS Program and DECLARE-TIMI 58 trials were not designed or powered to look at the patients with established $\mathrm{CV}$ disease and the high CV risk group separately. Hence, conclusions as drawn by the

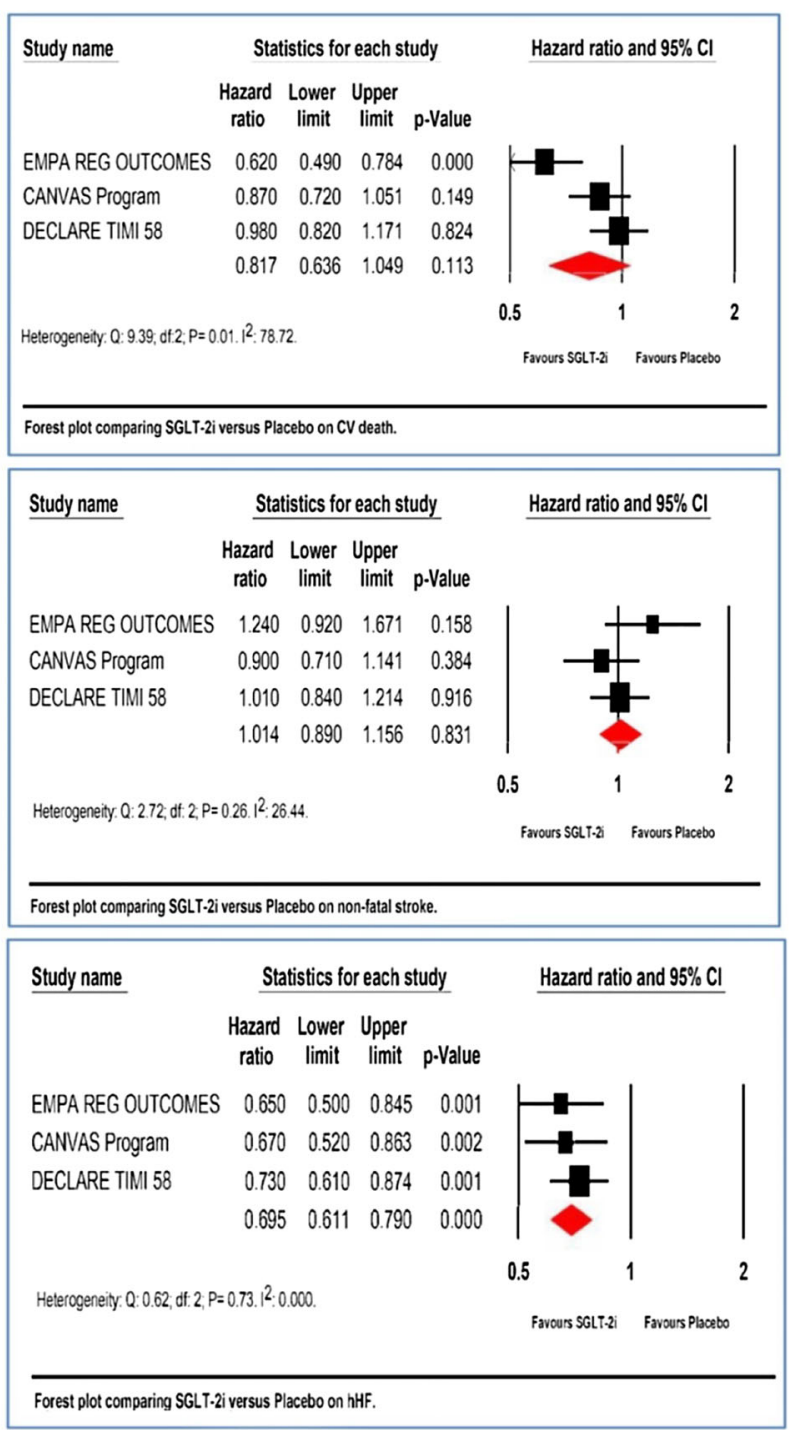

EMPA REG OUTCOMES: Empagliflozin. CANVAS program: canagliflozin. DECLARE TIMI 58: dapagliflozin

meta-analysis published in the Lancet should be drawn from the pooled data only and not from the established $\mathrm{CV}$ disease or high $\mathrm{CV}$ risk cohort separately, as was done in that publication. This meta-analysis studied the pooled data of all events and therefore sheds new insights into the supposed benefits of SGLT-2i on individual MACE events. Further insights into the $\mathrm{CV}$ outcomes aspect are expected with the publication of the VERTIS CV trial [13]. 


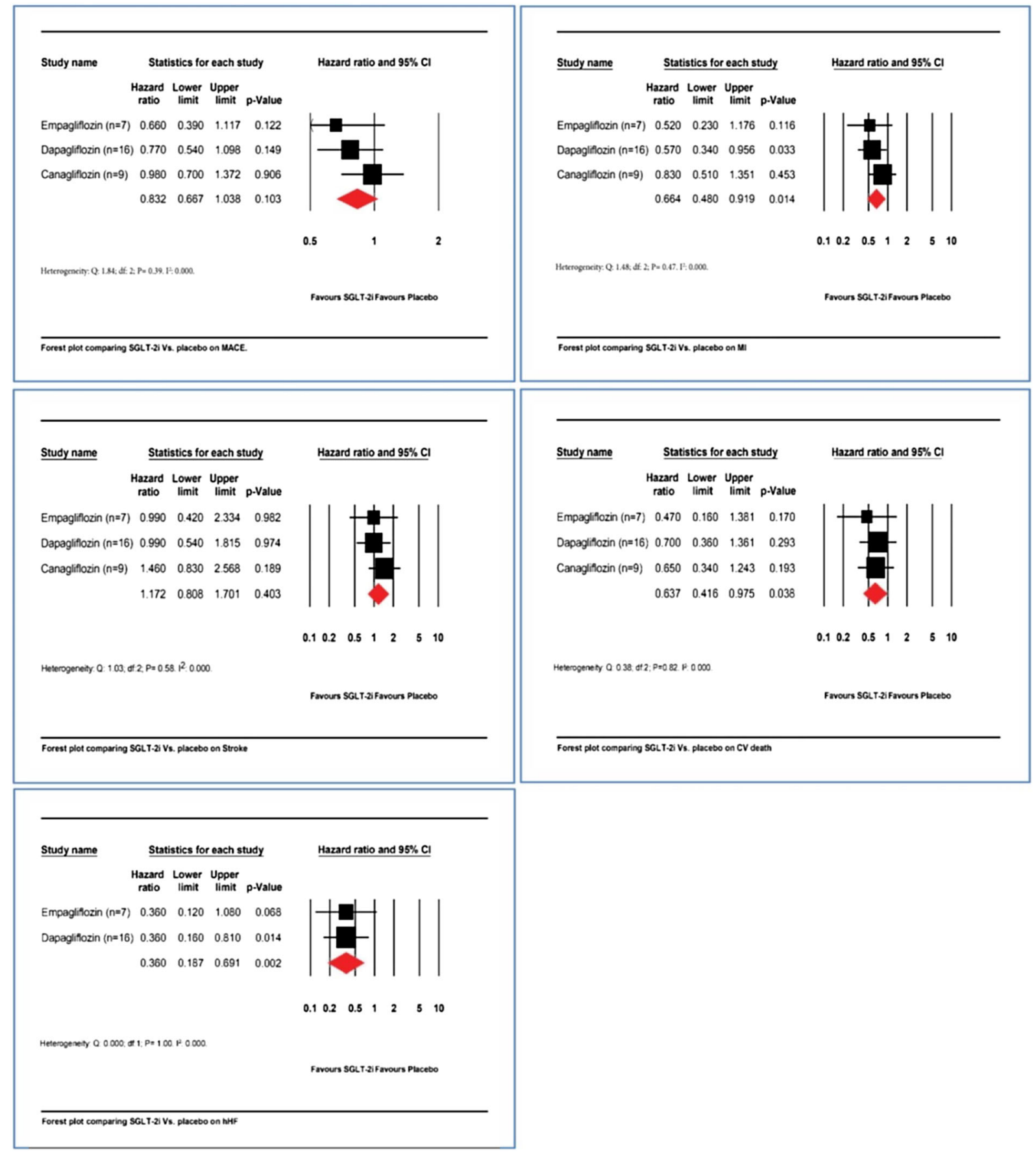

Fig. 4 CV effects of SGLT-2i versus placebo from pooled phase III data. a MACE; b MI; $\mathbf{c}$ stroke; $\mathbf{d}$ CV death; e hHF (canagliflozin not reported in pooled FDA analysis). $n$ number of phase III studies included

\section{Evidence from the Phase 3 Pooled Analysis}

With the meta-analyses of Zelniker et al. contrasting with this analysis, it was prudent to analyze the data from the approval phase, the events that occurred in the phase 3 trials with gliflozins pooled together. The results from these pooled data were a replica of the meta- 
analysis performed on the pooled CVOT with SGLT-2i. There were inconsistent results as far as the atherosclerotic end points were concerned (36\% reduction in $\mathrm{MI}$ and a $17 \%$ increased risk for stroke). In contrast, the CV death (36\% reduction) and hHF (64\% reduction) signals were consistent. The meta-analyses of both these independent cohorts complement each other.

\section{Evidence from Mechanistic Studies}

With the CVOTs and pooled phase 3 data not supporting a basis for altering the atherosclerotic cardiovascular disease burden, a number of hypotheses were sought to explain the positive $\mathrm{CV}$ effects. The CVOTs were not designed to answer these mechanistic queries, and post hoc analyses were conducted. In view of the fact that glycemic equipoise was achieved in all the CVOTs and BP (blood pressure), and weight reduction and natriuresis did not differ significantly with the placebo arm, these markers were unlikely to be the possible candidates for the observed CV benefits [14]. The elevation of blood ketones by SGLT-2i (the super fuel hypothesis) is viewed as the trigger for shifting the balance toward a more efficient fuel especially at times of stress, leading to positive $\mathrm{CV}$ outcomes, and is currently considered to be a plausible explanation for the benefits seen with these drugs [15]. The separation of the CV death and hHF curves within the first 3 months of start of the trials could only be explained by such metabolic factors. Another potential candidate is the elevated hematocrit level resulting in increased blood viscosity and its consequent impact on carotid wall shear stress [16]. Many type 2 diabetes patients have a background impaired ventricular repolarization. SGLT-2 inhibitors have been documented to improve QTc dispersion and hence restore the normal ventricular repolarization [17]. This could also explain the CV benefits associated with SGLT2i. However, SGLT-2i has a positive effect on endothelial dysfunction and arterial stiffness, defects that are very common in patients with type 2 diabetes, and this may be an obtuse route through which gliflozins might have an effect on the atherosclerotic process. However, that process would take years. The DEFENCE study demonstrated an improvement in flow-mediated dilation from baseline with SGLT-2i [18]. These are not necessarily sacrosanct markers for atherosclerosis. The mechanistic data as of now point toward an immediate, metabolic and electro-mechanical mechanism rather than a slow-paced impact on the atherosclerotic process, in keeping with the findings of this metaanalysis.

\section{Summary of Findings}

The analyses of CVOTs, pooled phase 3 trials and review of the mechanistic data indicate that the benefit of SGLT-2i is only restricted to the reduction of "hHF" and the "hHF or CV death" composite and not to reduction of $\mathrm{CV}$ death (only). Although there is a reduction in nonfatal MI, there is no reduction in non-fatal stroke, suggesting that the effects are restricted to the heart (pump) and not ASCVD (pipe). The significant reduction of $\mathrm{CV}$ death in the EMPAREG Outcomes trial alone was also possibly due to a reduction in hHF with the other significant contribution coming from death due to unassailable causes [19].

These findings are in keeping with the phase 3 and mechanistic data on SGLT-2i, which support that SGLT-2i probably works through metabolic and hemodynamic mechanisms, which in turn work on the myocardium to improve the pre- and after-load and also improve the myocardial energetic at the level of the "pump" [20].

\section{Study Limitations}

The inclusion of studies with a short duration might have curtailed the atherosclerotic cardiovascular benefits, if any. However, most of the available mechanistic and clinical data seem to indicate the cardiovascular benefits of SGLT$2 \mathrm{i}$ attributable to its hemodynamic and metabolic effects and not to the atherosclerotic burden. Another limitation was related to data analysis using the aggregate instead of individual patient data. 


\section{Strength of the Study}

The main strength of this analysis was the use of a pooled SGLT-2i analysis instead of analyzing underpowered subgroups. Moreover, the quality of studies included for analysis were of good quality with very low risk of bias and pooled end points devoid of significant heterogeneity. The conclusions from the data can be considered an impact of the entire class on cardiovascular end points and more unbiased than those of previous studies.

\section{CONCLUSION}

Contrasting the findings from the meta-analysis by Zelniker et al., this meta-analysis insists that since the DECLARE TIMI 58 and CANVAS Program studies were not adequately powered to assess the established and high CV-risk group separately, the results should be interpreted with caution. There is no evidence of any positive effect of SGLT-2i on the atherosclerotic end points (MI and stroke) from the individual studies, pooled phase 3 study analysis, mechanistic speculations or this meta-analysis on CVOTs. We would therefore like to exercise restraint as far as drawing any conclusion about the benefits of SGLT-2i on atherosclerotic CV disease. We firmly conclude that the predominant impact of SGLT-2i is on "hHF" and the "hHF or CV death" composite and not atherosclerotic CV disease, as suggested by Zelniker et al. (even in selected subgroups).

\section{ACKNOWLEDGEMENTS}

Funding. No funding or sponsorship was received for this study or publication of this article. The article processing charges were funded by the authors.

Authorship. All named authors meet the International Committee of Medical Journal Editors (ICMJE) criteria for authorship for this article, take responsibility for the integrity of the work as a whole and have given their approval for this version to be published.

Disclosures. Binayak Sinha and Samit Ghosal have nothing to disclose.

Compliance with Ethics Guidelines. This article is based on previously conducted studies and does not contain any studies with human participants or animals performed by any of the authors.

Data Availability. The data sets during and/ or analyzed during the current study are available from the corresponding author on reasonable request.

Open Access. This article is distributed under the terms of the Creative Commons Attribution-NonCommercial 4.0 International License (http://creativecommons.org/licenses/ by-nc/4.0/), which permits any noncommercial use, distribution, and reproduction in any medium, provided you give appropriate credit to the original author(s) and the source, provide a link to the Creative Commons license, and indicate if changes were made.

\section{REFERENCES}

1. Lambers Heerspink HJ, de Zeeuw D, Wie L, Leslie B, List J. Dapagliflozin a glucose-regulating drug with diuretic properties in subjects with type 2 diabetes. Diabetes Obes Metab. 2013;15:853-62.

2. Abdul-Ghani MA, Norton L, DeFronzo RA. Renal sodium-glucose cotransporter inhibition in the management of type 2 diabetes mellitus. Am J Physiol Ren Physiol. 2015;309:F889-900.

3. Levelt E, Mahmod M, Piechnik SK, Ariga R, Francis JM, Rodgers CT, et al. Relationship between left ventricular structural and metabolic remodelling in type 2 diabetes. Diabetes. 2016;65:44-52.

4. Pitt B, Zainad F, Remme WJ, Cody R, Castaigne A, Perez A, et al. The effect of spironolactone on morbidity and mortality in patients with severe heart failure. N Engl J Med. 1999;341:709-17.

5. Zinman B, Wanner C, Lachin JM, Fitchett D, Bluhmki E, Hantel S, et al. Empagliflozin, 
cardiovascular outcomes, and mortality in type 2 diabetes. N Engl J Med. 2015;373:2117-28.

6. Neal B, Perkovic V, Mahaffey KW, de Zeeuw D, Fulcher G, Erondu N, et al. Canagliflozin and cardiovascular and renal events in type 2 diabetes. N Engl J Med. 2017;377:644-57.

7. Wiviott SD, Raz I, Bonaca MP, Mosenzon O, Kato ET, Cahn A, et al. Dapagliflozin and cardiovascular outcomes in type 2 diabetes. NEJM 2018. [Online]. https://www.nejm.org/doi/full/10.1056/NEJMoa18 12389. Accessed 29 Nov 2018.

8. Zelniker TA, Wiviott SD, Raz I, Im K, Goodrich EL, Bonaca MP, et al. SGLT2 inhibitors for primary and secondary prevention of cardiovascular and renal outcomes in type 2 diabetes: a systematic review and meta-analysis of cardiovascular outcome trials. Lancet 2018. [Online]. https://www.thelancet.com/ journals/lancet/article/PIISO140-6736(18)32590-X/ fulltext. Accessed 1 Dec 2018.

9. Salsali A, Kim G, Woerle HJ, Broedl UC, Hantel S. Cardiovascular safety of empagliflozin in patients with type 2 diabetes: a meta-analysis of data from randomized placebo-controlled trials. Diabetes Obes Metab. 2016;18(10):1034-40.

10. Sonesson C, Johansson PA, Johnsson E, GauseNilsson I. Cardiovascular effects of dapagliflozinin patients with type 2 diabetes and different risk categories: a meta-analysis. Cardiovasc Diabetol. $2016 ; 15: 37$.

11. Clinical review division of metabolism and endocrinology products (DMEP). FARXIGA (Dapagliflozin). [Online]. https://www.accessdata.fda. gov/drugsatfda_docs/nda/2014/202293orig1s000ris kr.pdf. Accessed 1 Dec 2018.

12. Center for drug evaluation and research application number: 204042Orig1s000SUMMARY REVIEW. INVOKANA (Canagliflozin). [Online]. https://www. accessdata.fda.gov/drugsatfda_docs/nda/2013/2040 42orig1s000sumr.pdf. Accessed 4 Dec 2018.
13. Cannon CP, McGuire DK, Pratley R, Dagogo-Jack S, Mancuso J, Huyck S, et al. Design and baseline characteristics of the eValuation of ERTugliflozin effIcacy and Safety CardioVascular outcomes trial (VERTIS-CV). Am Heart J. 2018;206:11-23.

14. Abdul-Ghani M, Del Prato S, Chilton R, DeFronzo RA. SGLT2 inhibitors and cardiovascular risk: lessons learned from the EMPA-REGOUTCOME study. Diabetes Care. 2016;39:717-25.

15. Mudaliar S, Alloju S, Henry RR. Can a shift in fuel energetics explain the beneficial cardiorenal outcomes in the EMPA-REGOUTCOME study? A unifying hypothesis. Diabetes Care. 2016;39:1115-22.

16. Irace C, Casciaro F, Scavelli FB, Oliverio R, Cutruzzola A, Cortese C, et al. Empagliflozin influences blood viscosity and wall shear stress in subjects with type 2 diabetes mellitus compared with incretinbased therapy. Cardiovasc Diabetol. 2018;17:52.

17. Sato T, Miki T, Ohnishi H, Yamashita T, Takada A, Yano T, et al. Effect of sodium-glucose co-transporter-2 inhibitors on impaired ventricular repolarization in people with type 2 diabetes. Diabet Med. 2017;34:1367-71.

18. Shigiyama F, Kumashiro N, Miyagi M, Ikehara K, Kanda E, Uchino H, et al. Effectiveness of dapaglifozin on vascular endothelial function and glycemic control in patients with early-stage type 2 diabetes mellitus: DEFENCE study. Cardiovasc Diabetol. 2017;16:84.

19. FDA briefing document endocrine and metabolic Drug Advisory Committee meeting June 28, 2016. [Online]. https://www.fda.gov/downloads/Adviso ryCommittees/UCM508422.pdf. Accessed 4 Dec 2018.

20. Verma S, McMurray J, Cherney D. The metabolodiuretic promise of sodium-dependent glucose cotransporter 2 inhibition. The search for the sweet spot in heart failure. JAMA Cardiol. 2017;2(9):939-40. 